\title{
Titanium nitride: A new Ohmic contact material for $n$-type CdS
}

\author{
Arjen Didden, ${ }^{1, a)}$ Hemme Battjes, ${ }^{1}$ Raymond Machunze, ${ }^{2}$ Bernard Dam, ${ }^{1}$ \\ and Roel van de $\mathrm{Krol}^{1}$ \\ ${ }^{1}$ Department of Chemical Engineering, Materials for Energy Conversion and Storage, Delft University \\ of Technology, P.O. Box 5045, 2600 GA Delft, Netherlands \\ ${ }^{2}$ Department of Materials Science and Engineering, Delft University of Technology, Mekelweg 2, \\ 2628 CD Delft, Netherlands
}

(Received 4 May 2011; accepted 22 June 2011; published online 8 August 2011)

\begin{abstract}
In devices based on CdS, indium is often used to make Ohmic contacts. Since indium is scarce and expensive, suitable replacement materials need to be found. In this work, we show that sputtered titanium nitride forms an Ohmic contact with n-type CdS. The CdS films, deposited with chemical bath deposition, have a hexagonal crystal structure and are polycrystalline, mostly with a (002) texture. The thickness of the films is $\sim 600 \mathrm{~nm}$, and the donor density is $1.9 \times 10^{16} \mathrm{~cm}^{-3}$. The donor density increases to $1.5 \times 10^{17} \mathrm{~cm}^{-3}$ upon annealing. The contact resistivity of sputtered TiN on CdS is found to be $4.7 \pm 0.6 \Omega \mathrm{cm}^{2}$. This value is sufficiently small to avoid large resistive losses in most CdS device applications. To demonstrate the use of TiN in a CdS device, a $\mathrm{Au} / \mathrm{CdS} /$ TiN Schottky diode was constructed. The diode has a potential barrier of $0.69 \mathrm{~V}$ and an ideality factor of 2.2. @ 2011 American Institute of Physics. [doi:10.1063/1.3615946]
\end{abstract}

\section{INTRODUCTION}

$\mathrm{CdS}$ is an n-type semiconductor with a direct bandgap of $2.42 \mathrm{eV}^{1}$ that can be employed in a large variety of optoelectronic devices, such as highly efficient CIGS $^{2}$ and CdTe $^{3}$ solar cells and photodetectors, as well as gas sensors, ${ }^{4,5}$ field effect transistors, ${ }^{6,7}$ and LEDs. ${ }^{8}$ For most of these applications indium is used to form an Ohmic contact with CdS. However, since indium is a relatively scarce metal and at the same time a major component of transparent conducting oxides used in devices such as solar cells, touch screens, O-LEDs and flat panel displays, it is becoming increasingly expensive. This drives the search for alternative Ohmic contact materials that can replace indium.

We propose the use of titanium nitride (TiN) as an alternative for indium in n-type $\mathrm{CdS}$ devices. TiN is a well-known material that is both relatively low-cost and widely used in several applications, such as wear-resistant coatings on cutting tools, and $\mathrm{Cu}$ diffusion barriers in Si technology. Moreover, it is a material that can be deposited at temperatures as low as $60^{\circ} \mathrm{C}$ by metal-organic atomic layer deposition. ${ }^{9}$ This could make it a suitable contact material for applications based on CdS quantum dots, such as quantum dot solar cells, in which low-temperature processing is required to prevent the nanoparticles from sintering and losing their quantumsize effects. ${ }^{10}$

Reported work functions of TiN vary between 3.5 and $4.4 \mathrm{eV} .^{11,12}$ This is slightly lower than the reported electron affinities for $\mathrm{CdS}$, which range between 4.4 and $4.8 \mathrm{eV} .^{13,14}$ Based on these values, TiN is expected to form an Ohmic contact with n-type CdS. Obtaining an Ohmic contact in real devices is, however, not trivial. Due to Fermi-level pinning induced by interface defect states, Schottky-type potential barriers are often formed where Ohmic contacts would be

${ }^{\text {a)} E l e c t r o n i c ~ m a i l: ~ a . p . d i d d e n @ t u d e l f t . n l . ~}$ expected. This is illustrated by metals such as $\mathrm{Al}^{15,16}$ and $\mathrm{Zn},{ }^{17}$ which form a Schottky contact with n-type CdS despite their lower work functions. In this paper we investigate the electrical properties of the TiN/CdS contact, and show that an Ohmic contact is indeed formed.

\section{EXPERIMENTAL}

The CdS films were grown on FTO-coated glass (fluorine-doped tin dioxide, $15 \Omega / \square$, TEC 15 , Libbey-OwensFord) and glass substrates $(2 \times 3 \mathrm{~cm})$ by chemical bath deposition (CBD) ${ }^{18-20}$ The substrates were cleaned by ultrasonic rinsing in acetone and ethanol, followed by a $5 \mathrm{~s}$ dip in a dilute $\mathrm{HCl}$ solution $(17.5 \%)$ and subsequent rinsing with ultrapure de-ionized water (Milli-Q, $18.2 \mathrm{M} \Omega \mathrm{cm}$ ). After cleaning, the substrates were submerged in $60 \mathrm{ml}$ ultrapure water that was heated to $70^{\circ} \mathrm{C}$. CdS films were deposited by adding reactants from aqueous stock solutions (all prepared in ultrapure water) in the following order: $300 \mu \mathrm{l}$ of $0.5 \mathrm{M}$ $\mathrm{CdCl}_{2}$ hemi-pentahydrate (Aldrich, ACS reagent grade), $1320 \mu \mathrm{l} 2 \mathrm{M} \mathrm{NH}_{4} \mathrm{Cl}$ (JT Baker, 99.5\%), and $3800 \mu \mathrm{l}$ of 13.2 $\mathrm{M} \mathrm{NH}_{4} \mathrm{OH}$ solution (JT Baker). After 15 mins of homogenization with a magnetic stirrer, $1800 \mu \mathrm{l}$ of $1 \mathrm{M}$ thiourea (Aldrich, ACS reagent grade) was added slowly. After 60 mins, the samples were taken out of the bath and cleaned ultrasonically in ultrapure water for 30 seconds and rinsed with ultrapure water to remove loose particle deposits. Thicker layers were obtained by repeating the cycle of deposition, ultrasonic cleaning, and rinsing multiple times.

TiN contacts were deposited on CdS films and glass substrates by reactive unbalanced magnetron sputtering in an industrial PVD system (Hauzer HC 750). The substrate temperature was $200 \pm 20^{\circ} \mathrm{C}$. Prior to the deposition of TiN, the substrates were plasma-etched $\left(4 \times 10^{-3} \mathrm{mbar} \mathrm{Ar}\right)$ to remove impurities from the surface. The substrates performed a planetary motion in front of a $600 \times 120 \mathrm{~mm}^{2}$ titanium target; the 
nitrogen and argon flows during deposition were 37 and 115 sccm, respectively, yielding a deposition pressure of $4 \times 10^{-3}$ mbar. The base pressure of the system was $4 \times 10^{-5}$ mbar. The film was ion-bombarded during growth by applying a substrate bias voltage of $-125 \mathrm{~V}$ in order to increase the density. The target power was $5 \mathrm{~kW}$ and the target voltage was $600 \mathrm{~V}$, resulting in a deposition rate of $4.5 \mathrm{~nm} / \mathrm{s}$. Typical deposition times were $44 \mathrm{mins}$, resulting in $\sim 200 \mathrm{~nm}$ thick films. For comparison with the TiN contacts, Au Schottky contacts were deposited by thermal evaporation from a resistively heated tungsten boat in a home-built vacuum chamber having a base pressure of $10^{-7}$ mbar.

Film thickness values were measured with a Dektak 3 Profilometer. Grazing incidence X-ray diffraction spectra were measured with a Bruker D8 Advance diffractometer using $\mathrm{Cu}-\mathrm{K}_{\alpha}$ radiation and an incident angle of $0.9^{\circ}$.

Current-voltage measurements were carried out with an EG\&G 283 potentiostat (Princeton Applied Research). Voltage-dependent impedance measurements were carried out with a Solartron 1255 frequency response analyzer in combination with the EG\&G 283 potentiostat. The contact resistivity of the TiN/CdS contacts was measured with four-point probe measurements using a Keithley 2001 multimeter and the EG\&G 283 potentiostat, using spring-loaded gold contact pins.

\section{Texture of the CdS film}

The grazing incidence X-ray diffraction spectrum of the CdS film on a FTO substrate is shown in Fig. 1. Two phases, cubic and hexagonal, have been reported in the literature on chemical bath deposition of CdS. ${ }^{18,21-23}$ The diffraction pattern of our sample reveals a strong CdS peak at $26.7^{\circ}$ and a very small peak at $48^{\circ}$, which correspond to the (002) and (103) planes of hexagonal CdS, respectively. No traces of the cubic phase are observed. The diffraction pattern indicates a preferred growth direction with the c-axis oriented perpendicular to the surface. The same (002) orientation is found for films grown on uncoated glass substrates, and has also been observed by other authors. ${ }^{21-23}$ For hexagonal $\mathrm{CdS}$, a (002) orientation implies that the closest-packed lattice planes are exposed to the reaction mixture during growth. We attribute this to an atom-by-atom growth mechanism, as reported previously by Ortega-Borges et al. for

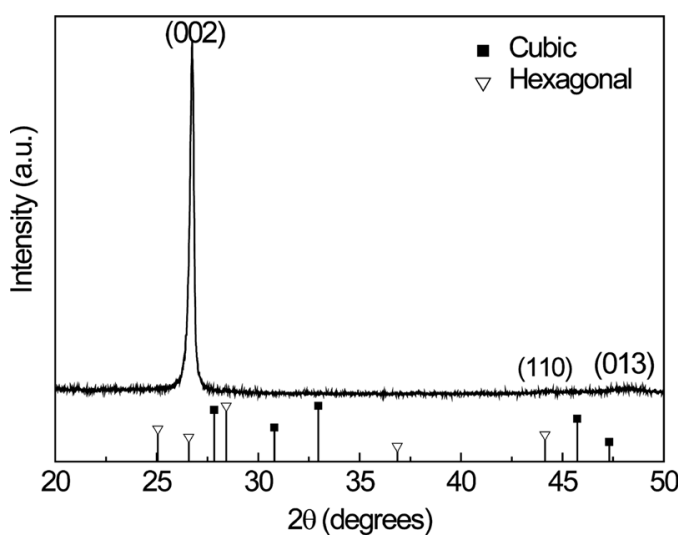

FIG. 1. Grazing incidence X-ray diffraction pattern of an as-deposited CdS film on a FTO substrate. chemical bath-deposited CdS. ${ }^{24}$ The reversible adsorption of ions from the solution allows the ions to find the energetically most favorable sites, analogous to surface diffusion processes that occur at sufficiently high substrate temperatures during physical vapor deposition (e.g., sputtering). ${ }^{25}$ The dynamic adsorption-desorption equilibrium in the CBD solution leads to recrystallization during film growth, and the growing film aims for the thermodynamically most stable configuration. Since the closest-packed lattice planes have the lowest surface energy, columnar growth with the (002) planes parallel to the surface will occur. This growth mechanism is analogous to the 'Type II' growth described by Mahieu et al. for sputter-deposited films. ${ }^{25}$ The deposited films have a clear and homogeneous appearance, which is indeed consistent with such a slow atom-by-atom growth. The thickness of the films after three deposition cycles was $\sim 600 \mathrm{~nm}$, which corresponds to $\sim 200 \mathrm{~nm}$ per cycle.

\section{The TiN/CdS contact}

To investigate the electrical properties of the TiN/CdS contact, circular TiN and Au contacts with diameters of 2 and $1 \mathrm{~mm}$, respectively, were deposited on the CdS film using a mask. The contacts were first deposited in a coplanar manner, as depicted in Figs. 2(a) and 2(b), to prevent shortcircuiting through pin-holes in the CdS film. The $J$ - $V$ curves of the $\mathrm{Au} / \mathrm{CdS} / \mathrm{TiN}$ system [Fig. 2(c)] reveal asymmetric diode-like behavior, indicating that one contact is blocking while the other one is Ohmic. Au has an electron work function of 5.3-5.5 $\mathrm{eV}^{1}$ and is well known to form a Schottky contact with $\mathrm{CdS} .^{26}$ The $J-V$ curve indeed confirms that the $\mathrm{CdS} / \mathrm{Au}$ Schottky junction is forward biased when a positive potential is applied to the Au contact. The TiN/CdS contact (a)

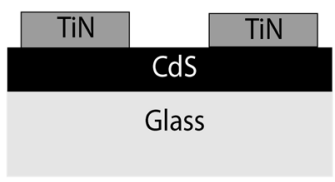

(c)

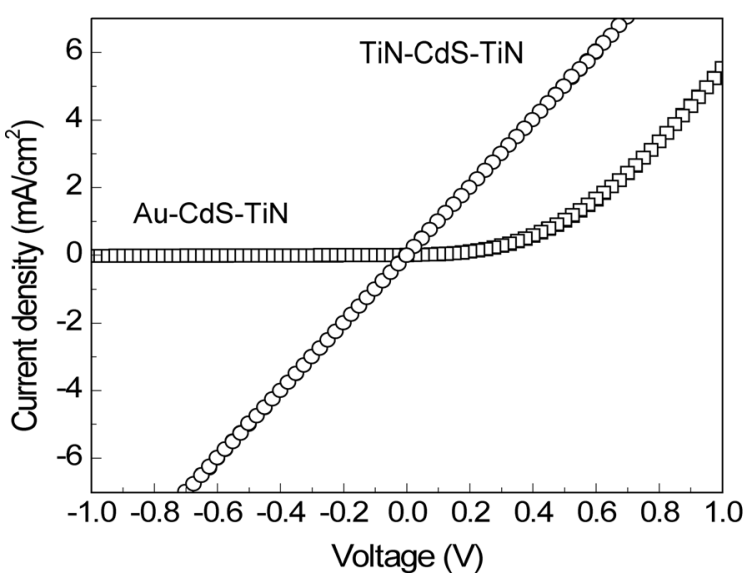

FIG. 2. Schematic representation of the lateral (coplanar) geometry of the $\mathrm{TiN} / \mathrm{CdS} / \mathrm{TiN}$ (a) and TiN/CdS/Au (b) device structures. (c) $J$ - $V$ curves of the $\mathrm{TiN} / \mathrm{CdS} / \mathrm{TiN}$ (open circles) and the $\mathrm{Au} / \mathrm{CdS} / \mathrm{TiN}$ (open squares) structures. 


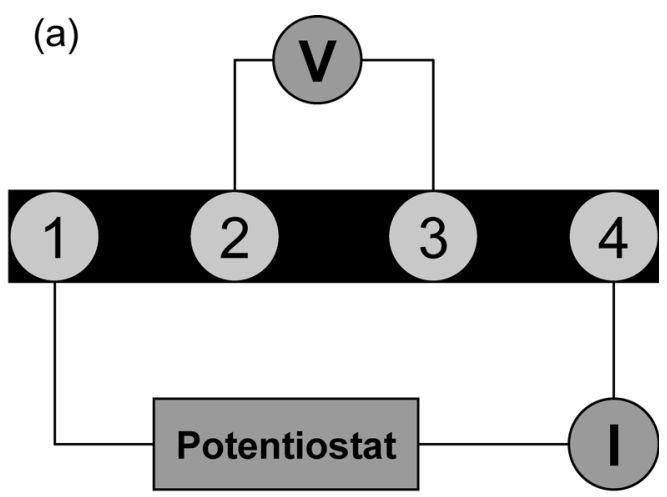

(b)

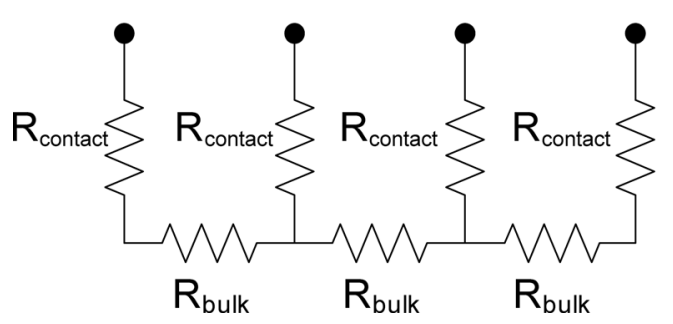

FIG. 3. (a) Layout of four-point resistance measurements. A current sent through points 1 and 4 gives rise to a potential difference between points 2 and 3 , from which the resistance $R_{14,23}\left(=V_{1,2} / I_{1,4}\right)$ is calculated. The rectangular dark-gray area is isolated from the surrounding material by cuts with a diamond scriber. (b) Equivalent circuit showing the bulk resistance and series resistance components.

must therefore be Ohmic in nature. This is indeed consistent with the fully Ohmic behavior that is observed when measuring between two TiN contacts, also shown in Fig. 2(c).

When TiN is to be used as Ohmic contact material for $\mathrm{CdS}$, both the bulk resistivity of the TiN layer and the contact resistance should be small enough not to cause a large voltage drop and adversely affect device performance. To determine the contact resistance, two-, three-, and four-point resistance measurements were carried out using the configuration shown in Fig. 3(a). The two-point resistance is given by e.g., $R_{12,12}=R_{\text {bulk }}+2 R_{\text {contact }}$, whereas the three-point resistance is given by $R_{13,12}=R_{\text {bulk }}+R_{\text {contact }}$. The contribution of $R_{\text {contact }}$ is negligible in the case of a four-point measurement. Comparing several sets of two-, three-, and four-point resistance measurements therefore allows us to determine $R_{\text {contact }}$. Measurements on a rectangular $\left(2 \times 17 \mathrm{~mm}^{2}\right)$ section of a $600 \mathrm{~nm} \mathrm{CdS} \mathrm{film} \mathrm{with} 2 \mathrm{~mm}$ TiN contacts yield a value of $R_{\text {contact }}=330 \pm 50 \Omega$. It should be noted that this value also includes contributions from the resistance between the gold pins and the TiN contacts, but separate measurements on a sputtered TiN film show this resistance to be negligible $(\sim 1.1 \Omega)$. The spreading resistance can also be neglected for these large contact areas. After normalizing $R_{\text {contact }}$ with respect to surface area, a value of $10 \pm 1.6 \Omega$ $\mathrm{cm}^{2}$ is found for the specific contact resistivity between TiN and CdS.

This value is several orders of magnitude higher than the contact resistivity reported for other TiN contacts on, for example, GaN and Si devices. ${ }^{27-29}$ The high value might be partly due to so-called 'current crowding' at the contacts, which means that only part of the TiN contact area is active in the transport of current from and to the CdS. This effect occurs because the diameter of the contact is much larger than the film thickness, and the current always chooses the path of the least resistance. ${ }^{30}$ While a detailed quantitative analysis is not possible with the current contact geometry, it is important to realize that the reported value of $10 \pm 1.6 \Omega$ $\mathrm{cm}^{2}$ represents an upper limit for $R_{\text {contact }}$ due to this effect.

To investigate the stability of the TiN/CdS contact, the sample was exposed to air for a period of 12 months. During this period, the contact resistivity increased by a factor of $\sim 3$ to $34 \pm 3 \Omega \mathrm{cm}^{2}$. The exact origin of the increased resistance is unknown, but the formation of a barrier layer by interdiffusion between TiN and CdS or oxidation of CdS via oxygen transport along grain boundaries perpendicular to the TiN/ $\mathrm{CdS}$ interface is a likely cause. Further optimization of the growth conditions may lead to fewer grain boundaries and improved stability in air, but this is beyond the scope of the present study.

\section{Mott-Schottky analysis of the CdS films}

Although the data in Fig. 2(c) as well as the four-point resistance measurements strongly suggest that the TiN/CdS contact is Ohmic, there is a possible alternative explanation for the apparent Ohmic behavior that has to be considered. The exposure of the CdS films to vacuum at high temperatures prior to TiN sputtering, and/or (re-)sputtering of CdS in the initial phase of TiN deposition may have caused a preferential removal of sulfur atoms from the CdS lattice. ${ }^{31}$ These sulfur vacancies act as electron donors and hence increase the concentration of free electrons in the CdS. Using the Kröger-Vink notation, this reaction can be written as follows:

$$
0 \leftrightarrow \frac{1}{2} S_{2}(g)+V_{S}^{\bullet \bullet}+2 e^{\prime} .
$$

At high sulfur vacancy concentrations, say $>10^{20} \mathrm{~cm}^{-3}$, the concomitantly high donor density results in a very narrow depletion layer. Tunneling of electrons through such a narrow depletion layer would result in apparent Ohmic behavior, even though a Schottky barrier is in fact present.

To investigate this possibility, the donor density of the CdS films has been determined from the space charge capacitance using impedance spectroscopy. Toward this end, Au contacts were evaporated on as-deposited and vacuumannealed CdS films. As shown previously, these contacts form Schottky barriers and a space charge layer is therefore formed in the $\mathrm{CdS}$ region close to the Au contact. FTO layers were used as an Ohmic back-contact, ${ }^{32}$ resulting in the parallel plate layout depicted in Fig. 4(a).

Figure 4(c) shows a Nyquist plot of the impedance of a vacuum-annealed FTO/CdS/Au sample. Similar data have been obtained for the as-deposited CdS films (not shown). The relation between the real $\left(Z^{\prime}\right)$ and the imaginary part of the impedance $\left(Z^{\prime \prime}\right)$ has the shape of a semicircle. The diameter of the circle depends strongly on the applied bias potential, which shows that the impedance is dominated by the space charge capacitance. The semicircle is slightly depressed, which indicates small deviations from the ideal 
(a)

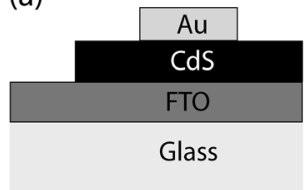

(c)

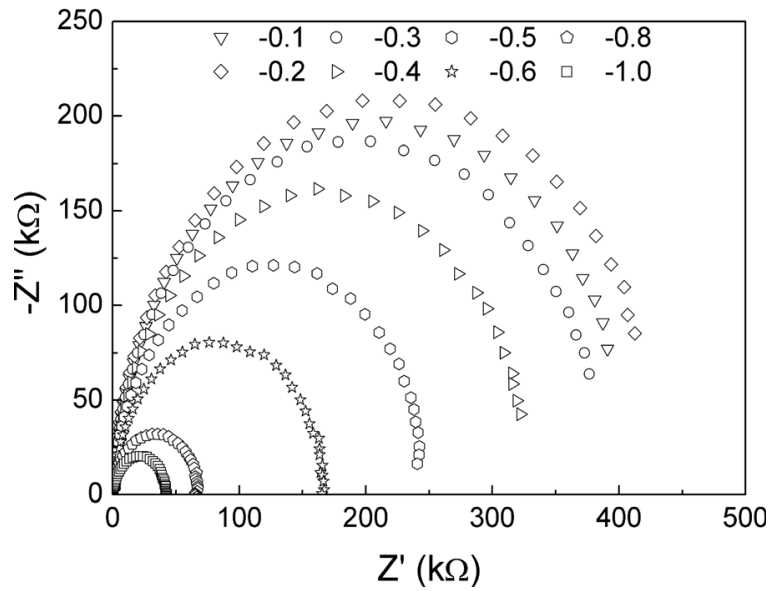

FIG. 4. (a) Layout of the impedance spectroscopy sample. (b) Equivalent circuit used to fit the impedance data, in which $R_{1}$ and $R_{2}$ are resistors and $\mathrm{CPE}$ is a constant phase element. (c) Nyquist plot of an FTO/CdS/Au sample annealed at $350^{\circ} \mathrm{C}$ in vacuum. The frequency ranges from $100 \mathrm{kHz}$ to 100 $\mathrm{Hz}$ in the clockwise direction.

capacitive behavior which we attribute to the presence of trap states in the $\mathrm{CdS}$ bulk or at the $\mathrm{CdS} / \mathrm{Au}$ interface. The data can be fitted using an equivalent circuit containing a series resistor $\left(R_{1}\right)$ and a resistor in parallel $\left(R_{2}\right)$ with a constant phase element (CPE), as illustrated in Fig. 4(b).

The complex impedance $Z$ of a constant phase element is given by

$$
Z=\frac{1}{Q(j \omega)^{n}}
$$

Here, $\omega$ is the angular frequency $(\mathrm{rad} / \mathrm{s})$, and $n$ is the nonideality factor of a CPE. For $n=1$, the CPE is an ideal capacitor with a capacitance $C=Q$, whereas the element is purely resistive for $n=0\left(R=Q^{-1}\right)$. For $0<n<1$, which is the case for a depressed semicircle, the equivalent capacitance of the CPE (with the dimensionally correct units of farads) can be calculated with ${ }^{33}$

$$
C=Q\left(\omega_{\max }\right)^{n-1} .
$$

This equation was used to convert the CPE values, obtained from the fitted impedance spectra, to actual space charge capacitance values. The average value of the non-ideality factor obtained from the fit is $0.97 \pm 0.01$.

Figure 5 shows the calculated space charge capacitances, plotted as $\left(1 / C_{S C}\right)^{2}$, as a function of the applied bias potential for an as-deposited and a vacuum-annealed $\mathrm{CdS}$ sample. From the slope of the line, the donor density $N_{D}$ of the CdS can be obtained using the Mott-Schottky equation ${ }^{34}$

$$
\left(\frac{A}{C}\right)^{2}=\left(\frac{2}{e \varepsilon_{0} \varepsilon_{r} N_{D}}\right)\left(V-\phi_{b i}-\frac{k T}{e}\right) .
$$

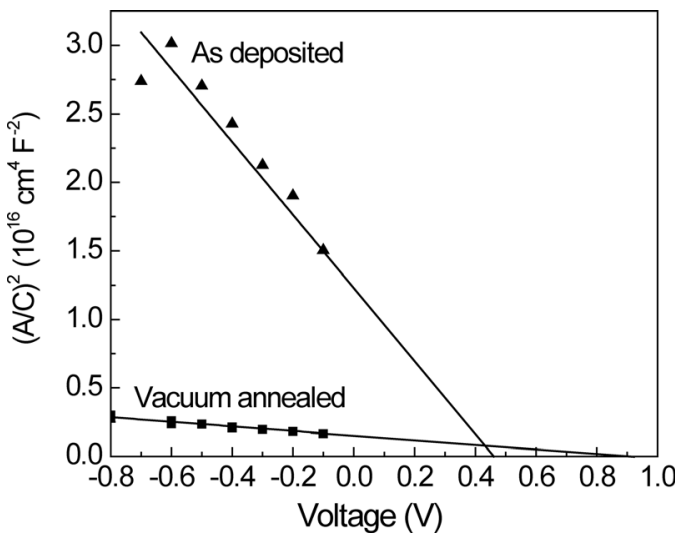

FIG. 5. Mott-Schottky plot for the Au/CdS junction of an as-deposited and a vacuum-annealed sample, with capacitance values obtained from the fits of the impedance spectra.

Here, $\varepsilon_{0}$ is the permittivity of vacuum $\left(8.854 \times 10^{-12} \mathrm{~F} / \mathrm{m}\right)$, $\varepsilon_{r}$ is the static dielectric constant $\left(8.7\right.$ for $\left.\mathrm{CdS}^{1}\right), \phi_{b i}$ is the built-in potential of the junction, $V$ is the applied bias potential, $A$ is the surface area, and all other symbols have their usual meaning. Donor density values of $1.9 \times 10^{16} \mathrm{~cm}^{-3}$ and $1.5 \times 10^{17} \mathrm{~cm}^{-3}$ are obtained for the as-deposited and vacuum-annealed samples, respectively. The donor density of the as-deposited sample is comparable to the values reported in the literature for CdS films deposited with CBD under similar conditions. ${ }^{23,35,36}$ The donor density of the vacuumannealed sample is an order of magnitude higher due to the loss of sulfur [cf. Eq. (1)]. To see if tunneling can play a role under these conditions, the corresponding width $w$ of the depletion layer at an applied bias of $0 \mathrm{~V}$, is calculated using the following expression: ${ }^{34}$

$$
w=\sqrt{\frac{2 \varepsilon_{0} \varepsilon_{r}\left(\phi_{b i}-\frac{k T}{e}\right)}{e N_{D}} .}
$$

For a donor density of $2 \times 10^{17} \mathrm{~cm}^{-3}$ and a typical built-in potential of $0.1-0.5 \mathrm{~V}$, a depletion layer width between 20 and $50 \mathrm{~nm}$ is found. Clearly, this rules out the possibility of tunneling through the depletion layer. This supports our assertion that TiN forms a true Ohmic contact with CdS.

It should be noted that there is a significant difference in the intercept with the voltage axis for the as-deposited and vacuum-annealed samples. Since the intercept with the voltage-axis is effectively the same as the built-in potential of the Schottky barrier $\left(\phi_{b i}\right)$, this means that the built-in potentials of the Schottky barriers are significantly different. The Schottky barrier height $\phi_{B n}$ can be calculated with $\phi_{B n}=\left(\phi_{b i}+\xi+k T / e\right){ }^{34}$ The parameter $\xi$ is the difference between the Fermi level and the conduction band in the semiconductor bulk, and is given by ${ }^{37}$

$$
\xi=k T \ln \left(\frac{N_{c}}{N_{D}}\right) .
$$

In this equation, $N_{c}$ is the effective density of states in the conduction band 


$$
N_{c}=2\left(\frac{2 \pi m_{e}^{*} k T}{h^{2}}\right)^{3 / 2}
$$

Extrapolation of the linear fits in Fig. 5 yields intercepts of $0.47 \pm 0.03$ and $0.91 \pm 0.01 \mathrm{~V}$. From the measured donor densities and an electron effective mass $m_{e}^{*}$ of $0.21 \times m_{0}{ }^{37}$ barrier heights of $0.59 \pm 0.03$ and $0.98 \pm 0.01 \mathrm{~V}$ are calculated for the as-deposited and vacuum annealed samples, respectively. Both these values are within the range of $0.2-$ $1.02 \mathrm{~V}$ reported for $\mathrm{Au} / \mathrm{CdS}$ Schottky diodes prepared with CBD. ${ }^{23,38,39}$ The smaller barrier height of the as-deposited sample is attributed to the presence of a second phase, possibly $\mathrm{CdO},{ }^{31}$ at the $\mathrm{Au} / \mathrm{CdS}$ interface. This phase forms an insulating layer that accommodates part of the work function difference between CdS and Au. The insulating layer is not present when the CdS is annealed under vacuum prior to depositing the Au contact. This means that the entire work function difference then falls across the space charge layer in the $\mathrm{CdS}$, which explains the larger values for the built-in potential and barrier voltage. The exact nature of the insulating layer is unknown, but it either has a high enough vapor pressure to evaporate during vacuum annealing, or the vacuum anneal increases its conductivity to a degree that it cannot sustain a significant voltage drop. Due to its negligible vapor pressure at $350{ }^{\circ} \mathrm{C},{ }^{40} \mathrm{CdO}$ is unlikely to evaporate, but its (n-type) conductivity is indeed likely to increase through oxygen loss during a vacuum anneal at this temperature.

The donor density values found above can be used to estimate the degree of non-stoichometry of the CdS. Here, we assume that sulfur vacancies are the main source of electron donors. The degree of ionization can be calculated with ${ }^{37}$

$$
\frac{N_{D}^{+}}{N_{D}}=\frac{1}{1+g_{D} \exp \left(\frac{E_{F}-E_{D}}{k T}\right)} .
$$

The donor level $E_{D}$ of the doubly-ionized sulfur vacancy $\left(V_{S}^{\bullet \bullet}\right)$ is located $0.445 \mathrm{eV}$ below the conduction band edge. ${ }^{21}$ For ntype CdS with an (ionized) donor density of $1.9 \times 10^{16} \mathrm{~cm}^{-3}$, the Fermi level at room temperature is $0.12 \mathrm{eV}$ [cf. Eq. (6)] below the conduction band, so $\left(E_{F}-E_{D}\right)=0.325 \mathrm{eV}$. The degeneracy factor $g_{D}$ has the standard value of two. ${ }^{37}$ This results in an ionization degree of $0.83 \%$ for the as-deposited sample, indicating that the concentration of sulfur vacancies is $1.9 \times 10^{16} / 0.0083=2.3 \times 10^{18} \mathrm{~cm}^{-3}$. This corresponds to approximately $0.01 \%$ of the total number of sulfur ions, indicating that these chemical bath-deposited films are highly stoichometric. $^{41}$

\section{Au/TiN/CdS diode}

Now that a high donor concentration in the CdS can be ruled out as a possible cause for the observed Ohmic nature of the TiN/CdS contact, we turn our attention to the CdS/ TiN interface. To investigate the possibility that sputterinduced damage in the CdS is responsible for the Ohmic contact behavior of the TiN/CdS junction, CdS films were deposited on top of the TiN films with CBD. With this configuration the presence of sputter-induced damage in $\mathrm{CdS}$

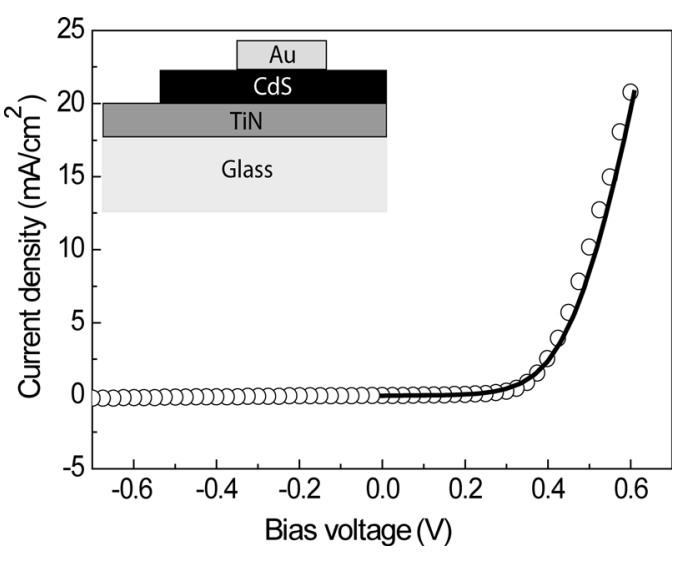

FIG. 6. I-V curve of an Au/CdS/TiN diode, with the Au contact connected as the working electrode of the device. The open squares are the measured values, and the solid line is a best fit of the current to Eq. (10). The inset of the graph shows the layout of the sample with parallel contacts.

near the TiN/CdS interface can be excluded. Moreover, since $\mathrm{TiN}$ is less prone to oxidation than $\mathrm{CdS}$, adverse effects of an oxide interface layer can be minimized. After CBD, the $\mathrm{CdS}$ film was annealed at $250{ }^{\circ} \mathrm{C}$ in vacuum. The sample was then quickly transferred to the evaporation chamber to minimize oxidation and $\mathrm{Au}$ contacts were evaporated onto the CdS film to create a Schottky contact. The structure of this $\mathrm{Au} / \mathrm{CdS} / \mathrm{TiN}$ Schottky diode is given in the inset of Fig. 6 . The current-voltage behavior of this structure, shown in Fig. 6, reveals that a non-blocking (Ohmic) contact is again formed at the TiN/CdS junction. This clearly demonstrates that sputter damage does not affect the behavior of the junction, and that it is inherently Ohmic in nature.

Even though the $I-V$ curve of the $\mathrm{Au} / \mathrm{CdS} / \mathrm{TiN}$ diode presented in Fig. 6 shows a clear diode-like behavior, it does not follow the ideal exponential behavior expected for a Schottky diode. ${ }^{34}$ At low currents, the curve is approximately exponential, but as the current increases, the behavior starts to become linear. This indicates a resistive component in series with the $\mathrm{Au} / \mathrm{CdS}$ Schottky junction that causes a voltage drop that is comparable to the diode voltage drop. This means that

$$
V=\Delta V_{\text {diode }}+\Delta V_{\text {resistor }}
$$

The voltage drop over the diode can be calculated rewriting the standard expressions for the $J-V$ characteristics of a Schottky diode ${ }^{34}$ and Ohm's law and rewriting it for forward bias

$$
V=\frac{n k T}{e} \ln \left(\frac{J+J_{0}}{J_{0}}\right)+J R .
$$

Here, $n$ is the diode's dimensionless non-ideality factor. The saturation current $J_{0}$ is given by ${ }^{34}$

$$
J_{0}=A^{*} T^{2} \exp \left(\frac{-e \phi_{B n}}{k T}\right) .
$$

The value of the effective Richardson constant $A^{*}$ is $120 \times\left(m^{*} / m_{0}\right) \mathrm{A} / \mathrm{cm}^{2} \mathrm{~K}^{2}$, which yields a value of $23 \mathrm{~A} / \mathrm{cm}^{2}$ $\mathrm{K}^{2}$ using $m^{*}=0.19 \times m_{0}$ for $\mathrm{CdS} .{ }^{39,42}$ 


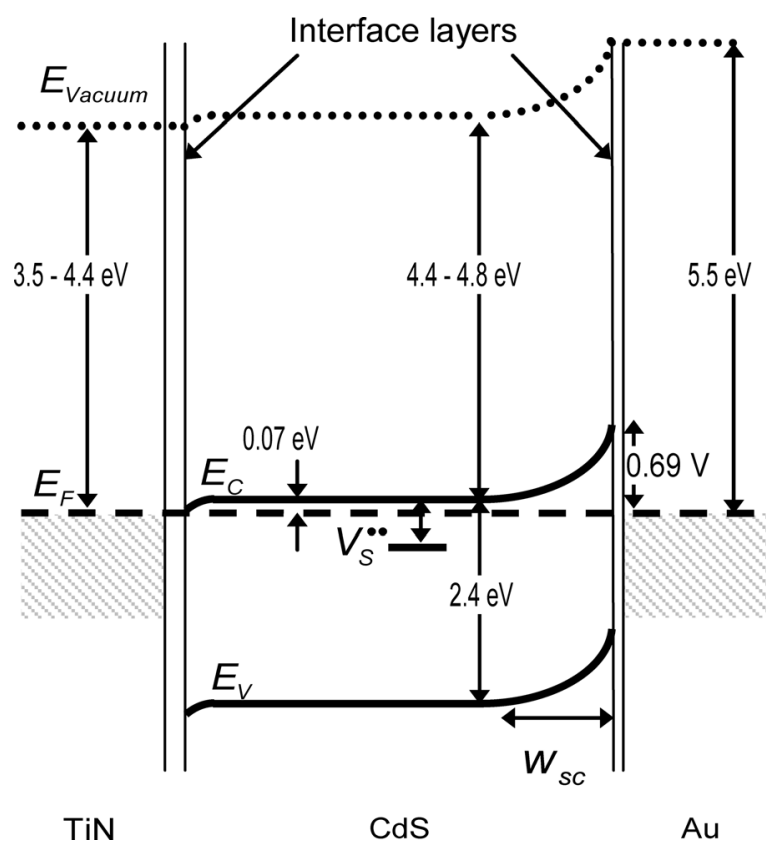

FIG. 7. Proposed band diagram of the Au/CdS/TiN Schottky diode under equilibrium conditions.

The barrier height, series resistance and the non-ideality factor have been obtained from the fit of the data. The barrier height is $0.69 \pm 0.01 \mathrm{~V}$, which is in the range of values obtained from the Mott-Schottky measurements. The nonideality factor is $2.2 \pm 0.1$, which is in good accordance with values of Schottky diodes made from nanocrystalline CdS films. ${ }^{39,43,44}$ The series resistance obtained from the fit is $149 \pm 18 \Omega$, which represents the sum of the bulk CdS resistance and the contact resistance of the TiN/CdS contact. Because the distance between the Au contact and the TiN back contact is only a few hundred nanometers, the magnitude of the bulk resistance is negligible. This implies that the value of the series resistance is approximately equal to the contact resistance, which leads to a contact resistivity of $4.7 \pm 0.6 \Omega \mathrm{cm}^{2}$. This is a factor of $\sim 2$ lower than the upperlimit value determined for the lateral contact configuration.

To summarize the findings, a band diagram of the $\mathrm{Au} /$ CdS/TiN Schottky diode is proposed in Fig. 7.

\section{CONCLUSIONS}

We have demonstrated that sputtered TiN forms an Ohmic contact with n-type $\mathrm{CdS}$ made by chemical bath deposition. This is in accordance with the expectations based on the work functions of both materials. The existence of a semi-Ohmic contact in the form of a narrow Schottky barrier through which electrons can tunnel, could be ruled out explicitly. A value of $4.7 \pm 0.6 \Omega \mathrm{cm}^{2}$ has been found for the contact resistivity of a sputtered TiN contact on n-type CdS. This implies that the Ohmic voltage loss for a standard current density of $10 \mathrm{~mA} / \mathrm{cm}^{2}$ is less than $50 \mathrm{mV}$, which is acceptable for most applications. We have demonstrated the feasibility of TiN as an Ohmic contact material for CdS by having fabricated a TiN/CdS/Au Schottky diode. This diode showed an ideality factor of 2.2 and a Schottky barrier height between 0.6 and $1.0 \mathrm{~V}$, depending on the treatment of the $\mathrm{CdS}$ prior to depositing the Au Schottky contact.

\section{ACKNOWLEDGMENTS}

This research is financially supported by the Thin Film Nanomanufacturing program of the Dutch Technology Foundation STW (project 10016). The authors thank W.F.A. Besling (NXP Semiconductors, Eindhoven, The Netherlands) and D.E. Nanu (AST BV., Leeuwarden, The Netherlands) for stimulating discussions.

${ }^{1}$ W. M. Haynes, ed., CRC Handbook of Chemistry and Physics, 91st Edition (Internet Version 2011), CRC Press/Taylor and Francis, Boca Raton, FL.

${ }^{2}$ I. Repins, M. A. Contreras, B. Egaas, C. DeHart, J. Scharf, C. L. Perkins, B. To, and R. Noufi, Progress in Photovoltaics 16(3), 235 (2008).

${ }^{3}$ D. Cunningham, K. Davies, L. Grammond, E. Mopas, N. O'Connor, M. Rubcich, M. Sadeghi, D. Skinner, T. Trumbly, Conference Record of the Twenty-Eighth IEEE Photovoltaic Specialists Conference, 2000, 13 (2000).

${ }^{4}$ N. Golovan and V. Smyntyna, Sensors and Actuators B-Chemical 6(1-3), 289 (1992).

${ }^{5}$ B. K. Miremadi, K. Colbow, and Y. Harima, Review of Scientific Instruments 68, 3898 (1997).

${ }^{6}$ X. F. Duan, C. M. Niu, V. Sahi, J. Chen, J. W. Parce, S. Empedocles, and J. L. Goldman, Nature 425(6955), 274 (2003).

${ }^{7}$ F. Y. Gan and I. Shih, IEEE Transactions on Electron Devices 49(1), 15 (2002).

${ }^{8}$ T. Ota, K. Takahash, and K. Kobayash, Solid-State Electronics 15(12), 1387 (1972).

${ }^{9}$ J. W. Elam, M. Schuisky, J. D. Ferguson, and S. M. George, Thin Solid Films 436(2), 145 (2003).

${ }^{10}$ E. Talgorn, R. D. Abellon, P. J. Kooyman, J. Piris, T. J. Savenije, A. Goossens, A. J. Houtepen, and L. D. A Siebbeles, Acs Nano 4(3), 1723 (2010).

${ }^{11}$ B. P. Luther, S. E. Mohney, and T. N. Jackson, Semiconductor Science and Technology 13(11), 1322 (1998).

${ }^{12}$ F. Fillot, T. Morel, S. Minoret, I. Matko, S. Maitrejean, B. Guillaumot, B. Chenevier, and T. Billon, Microelectronic Engineering 82(3-4), 248 (2005).

${ }^{13}$ J. Fritsche, D. Kraft, A. Thissen, T. Mayer, A. Klein, and W. Jaegermann, Thin Solid Films 403, 252 (2002).

${ }^{14}$ R. K. Swank, Phys. Rev. 153(3), 844 (1967).

${ }^{15}$ S. Gupta, D. Patidar, N. S. Saxena, and K. Sharma, Chalcogenide Letters 6(12), 723 (2009).

${ }^{16}$ A. A. M. Farag, I. S. Yahia, and M. Fadel, International Journal of Hydrogen Energy 34(11), 4906 (2009).

${ }^{17}$ S. Gupta, D. Patidar, N. S. Saxena, K. Sharma, and T. P. Sharma, Optoelectronics and Advanced Materials-Rapid Communications 2(4), 205 (2008).

${ }^{18}$ H. Khallaf, I. O. Oladeji, G. Y. Chai, and L. Chow, Thin Solid Films $\mathbf{5 1 6}(21), 7306$ (2008).

${ }^{19}$ I. O. Oladeji, L. Chow, J. R. Liu, W. K. Chu, A. N. P. Bustamante, C. Fredricksen, and A. F. Schulte, Thin Solid Films 359(2), 154 (2000).

${ }^{20}$ I. O. Oladeji and L. Chow, J. Electrochem. Soc. 144(7), 2342 (1997).

${ }^{21}$ K. S. Ramaiah, R. D. Pilkington, A. E. Hill, R. D. Tomlinson, and A. K. Bhatnagar, Mat. Chem. Phys. 68(1-3), 22 (2001).

${ }^{22}$ M. D. Archbold, D. P. Halliday, K. Durose, T. P. A. Hase, D. SmythBoyle, and K. Govender, Conference Record of the Thirty-First IEEE Photovoltaic Specialists Conference 2005, 476 (2005).

${ }^{23}$ N. B. Chaure, S. Bordas, A. P. Samantilleke, S. N. Chaure, J. Haigh, and I. M. Dharmadasa, Thin Solid Films 437(1-2), 10 (2003).

${ }^{24}$ R. Ortegaborges and D. Lincot, J. Electrochem. Soc. 140(12), 3464 (1993).

${ }^{25}$ S. Mahieu, P. Ghekiere, D. Depla, and R. De Gryse, Thin Solid Films 515, 1229 (2006)

${ }^{26}$ W. G. Spitzer and C. A. Mead, J. Appl. Phys. 34(10), 3061 (1963).

${ }^{27}$ S. Gautier, P. Komninou, P. Patsalas, T. Kehagias, S. Logothetidis, C. A. Dimitriadis, and G. Nouet, Semiconductor Science and Technology 18(6), 594 (2003).

${ }^{28}$ J. Hu, M. Ameen, G. Leusink, D. Webb, and J. T. Hillman, Thin Solid Films 308, 589 (1997)

${ }^{29}$ A. Paranjpe and M. Islamraja, J. Vac. Sci. Tech. B 13(5), 2105 (1995). 
${ }^{30}$ D. K. Schröder, Semiconductor Material and Device Characterization, 2 ed. (John Wiley \& Sons Inc., New York, 1998), p. 149-150.

${ }^{31}$ D. W. Niles, G. Herdt, and M. Al Jassim, J. Appl. Phys. 81(4), 1978 (1997).

${ }^{32}$ D. Lincot and R. O. Borges, J. Electrochem. Soc. 139(7), 1880 (1992).

${ }^{33}$ C. H. Hsu and F. Mansfeld, Corrosion 57(9), 747 (2001).

${ }^{34}$ E. H. Rhoderick and R. H. Williams, Metal-Semiconductor Contacts, 2 ed. (Oxford University Press, Oxford, 1988), pp. 38.

${ }^{35}$ M. E. Ozsan, D. R. Johnson, M. Sadeghi, D. Sivapathasundaram, G. Goodlet, M. J. Furlong, L. M. Peter, and A. A. Shingleton, J. Mater. Sci.-Mater. Electr. 7(2), 119 (1996).

${ }^{36}$ H. Chavez, M. Jordan, J. C. McClure, G. Lush, and V. P. Singh, J. Mater. Sci.-Mater. Electron. 8(3), 151 (1997).

${ }^{37}$ S. M. Sze, Physics of Semiconductor Devices, 2 ed. (John Wiley \& Sons, New York, 1981).
${ }^{38}$ S. K. Mandal, A. B. Maity, J. Dutta, R. Pal, S. Chaudhuri, and A. K. Pal, Physica Status Solidi A-Applied Research 163(2), 433 (1997).

${ }^{39}$ B. K. Patel, K. K. Nanda, and S. N. Sahu, J. Appl. Phys. 85(7), 3666 (1999).

${ }^{40}$ R. H. Lammoreaux, D. L. Hildebrand, and L. Brewer, J. Phys. Chem. Ref. Data 16(3), 419 (1987).

${ }^{41}$ Due to charge neutrality, oxygen atoms at anion sites $\left(\mathrm{O}_{\mathrm{S}}{ }^{\mathrm{x}}\right)$ are not taken into account in this equation.

${ }^{42}$ P. E. Lippens and M. Lannoo, Phys. Rev. B 39(15), 10935 (15-5-1989).

${ }^{43}$ H. P. Maruska, F. Namavar, and N. M. Kalkhoran, Appl. Phys. Lett. 61(11), 1338 (1992).

${ }^{44}$ S. K. Mandal, A. B. Maity, J. Dutta, R. Pal, S. Chaudhuri, and A. K. Pal, Physica Status Solidi A-Applied Research 163(2), 433 (1997). 